\title{
In Memoriam, HE Professor Dr. Christopher Gregory Weeramantry ${ }^{1}$
}

\author{
Sergio Peña Neira \\ sergio.penaneira@gmail.com \\ Profesor Asociado de Derecho internacional público y Filosofía del Derecho en la Universidad Bernardo \\ O’Higgins, Doctor por la Universidad Internacional de Andalucía.
}

El día 5 de enero de 2017 ha fallecido en Colombo, Sri Lanka, uno de los juristas más relevantes para el Derecho internacional público y particularmente para el Derecho internacional del Desarrollo, el profesor Cristopher G. Weeramantry. Nacido el 17 de noviembre de 1926 en Colombo, Ceylán gran parte de su trabajo lo efectuó allí, en Australia y en La Haya (Holanda).

El Centro de Estudios de Derecho Internacional Sustentable (en sus siglas en inglés CISDL) ha expresado que el juez Weeramantry, como se le conoció universalmente, fue uno de sus miembros más ilustres. Desarrolló, sin duda, un trabajo extraordinariamente fecundo en cuanto a sus ideas desde su trabajo como juez. Un jurista de gran bondad y autoexigencia. Fue uno de sus fundadores y patrón del mismo por 16 años. $^{2}$

Sus estudios los realizó en Sri Lanka, Universidad de Ceylan, su país natal, en aquel tiempo el Royal College, Colombo para luego dedicar su tiempo y esfuerzo en una licenciatura en Derecho y doctorado en el King's College London, una universidad de fama mundial, hoy parte de la Universidad de Londres. Esta universidad, sin duda, es la más importante universidad formadora de juristas en derecho internacional, transnacional, filosofía jurídica y derecho tributario del mundo. Sus estudios en Sri Lanka fueron excelsos en cuanto obtuvo premios de diversa índole. ${ }^{3}$ Sus primero pasos se encaminaron a la Historia para luego, bajo la influencia de su hermano Lucien Weeramantry, dedicarse al Derecho. ${ }^{4}$

1 Artículo enviado el 13.07.2017 y aceptado el 19.07.2017.

El autor agradece a A. M. A. Wasantha Kumara, juez del Poder Judicial de Sri Lanka y Coordinador Académico del Instituto de Jueces de Sri Lanka. Asimismo, recordar que ha sido citado permanentemente por el Profesor Raúl Campusano D.

2 Marie Claire Cordonnier-Segger, Message from CISDL Senior Director on the Passing of HE Judge CG Weramantry, CISDL, Montreal, 2017.

3 "Judicial Service Association of Sri Lanka", Nensletter of the Judicial Service Association of Sri Lanka (2017), vol. 1, 9.

4 Kalana Senaratne, "Judge CG Weeramantry-of memories and memoirs yet unpublished", The Island Online, s.f. http://www.island.lk/2008/11/23/features4.html

Número de página no utilizable para citar

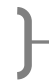


Se casó con Rosemary de Sampayo en $1959 .^{5}$

Su carrera como abogado se prolongó desde 1948, año en que fue llamado al Colegio de Abogado (se le otorgó licencia de ejercicio por dicho colegio luego de haber aprobado los exámenes correspondientes) hasta 1967 cuando fue llamado como juez a la Corte Suprema de Ceylán. En Ceylán escribió varios muy interesantes libros que expresan sus ideas desarrolladas en la Jurisprudencia durante su cargo como Juez en la Corte Internacional de Justicia. En Derecho de los Contratos, su primera área de trabajo, escribió "The Law of Contracts: A Comparative Study of the Roman-Dutch, English and Customary Laws of Contract in Ceylon (2 Volúmenes" y luego, en Derecho internacional derivó al área de Derecho internacional y teoría o filosofía jurídica pudiendo contarse, entre sus primeros trabajos, "Law: The Threatened Peripheries" y "An invitation to the Law".

En 1972 se retiró de dicha posición y asumió la calidad de profesor de la Universidad Monash en Australia como "Sir Hayden Starke Professor of Law". Según se indica la calidad de Juez de la Corte Suprema de Sri Lanka lo hubiere llevado a convertirse en el Presidente de dicha corte en razón de quienes lo precedían en calidad hubieren debido jubilarse. ${ }^{6}$ Su más interesante cargo, desde donde ha sido vastamente conocido, fue como juez de la Corte Internacional de Justicia nombrado en 1991 y cuyo término en dicha calidad se planteó en 2000, no sólo por su participación en dicha calidad de diversos casos famosos sino, como es lógico, en haber dado su opinión en los mismos. Fungió como Vicepresidente de dicho tribunal entre 1997 a 2000.

El primer caso en que entregó su opinión individual fue en la "Aplicación de la Convención sobre prevención y castigo del Crimen de Genocidio” (Bosnia y Herzegovina vs. Serbia y Montenegro)" cuya sentencia fue dictada el 11 de julio de 1996. Sin embargo, había participado en las sentencias sobre "Caso concerniente a las plataformas (Islamic Republic of Iran v. United States of America) (Objeciones preliminares)" de 12 de diciembre de 1986 donde rechazó la excepción de los Estados Unidos de incompetencia de la Corte conforme al Tratado de 1955 y que poseía competencia conforme al artículo XXI del mismo tratado.

Su opinión más famosa la constituye la relacionada al tema del uso o amenaza de uso de armas nucleares, una Opinión disidente en el caso del mismo nombre que fuere remitido por la Asamblea General de las Naciones Unidas como Opinión Consultiva de 1995 resuelta en 1996. Este caso le permitió al Juez Weeramantry, como se lee en una entrevista en 2007, indicar a sus colegas jueces que no cabía discusión o apertura en la posibilidad del uso o amenaza de este tipo de armas. Así indicó que "[1]a mayoría de los jueces de la Corte Internacional de Justicia no resolvieron en favor de la legalidad del uso de armas nucleares en legítima defensa cuando la supervivencia del Estado estuviere en entredicho. Lo que ellos hicieron fue dejar la esta pregunta abierta sin una determinación en un sentido u otro y ausente de afirmar la ilegalidad de las armas

Judicial Service Association of Sri Lanka, 9.

Judicial Service Association of Sri Lanka, 9.

Número de página no utilizable para citar 
nucleares en cualquier otra circunstancia. Mi desacuerdo con ellos es que no quise dejar posibilidad en cualquier circunstancia para el uso de las armas nucleares."7 Es más, así inicia el Profesor Weeramantry su opinión en calidad de Juez de la Corte Internacional de Justicia en su Opinión Disidente: "Mi opinión considerada es que el uso o la amenaza del uso de armas nucleares es ilegal en cualquier circunstancias sin excepción."8

Otro caso de gran importancia donde nuestro autor desarrolló su talento e inteligencia fue el caso "Gabčíkovo-Nagymaros Project (Hungary/Slovakia)". En este fallo, este gran disidente, del mismo modo que el Juez chileno Alejandro Alvarez Jofré, vastamente criticado por sus opiniones disidentes, desarrolló conceptos novedosos sobre ciertas materias. La primera, el desarrollo sustentable, fue objeto de su atención. Esta materia había sido esbozada y planteada por Alvarez en el célebre caso de Africa SudOeste. ${ }^{9}$ El principio de "continuidad de la Evaluación de Impacto Ambiental" es el segundo tópico tratado en dicha opinión disidente. Asimismo, finalmente trató las obligaciones erga omnes en los procedimientos judiciales inter partes. En el primer tema, desarrollo sostenible, consideró que no sólo el daño ambiental debía ser considerado en el fallo sino el desarrollo sustentable del país demandado, en el caso, Eslovaquia. Allí enuncia el "Principio de desarrollo sustentable". ${ }^{10}$ En ella, además, se insinúa la idea de "balance" entre las "consideraciones ambientales" y las "consideraciones de desarrollo". " Considera que es no sólo un "concepto" sino un "principio cuyo valor normativo es crucial para la decisión del caso". ${ }^{12}$ Indica que se enfrentan dos principios, el derecho al desarrollo y el derecho a la protección del medio ambiente: una colisión de derechos cuyo titular es el Estado. ${ }^{13}$ En la solución del problema, generación de electricidad a través de construcción de represas productoras de la misma versus destrucción de flora y fauna, aparece como solución, según el autor de la Opinión Disidente, "El principio es el Principio del Desarrollo Sostenible y en mi visión es parte integral del moderno Derecho internacional." ${ }^{\prime 14}$ El problema principal, jurídicamente hablando, es el de la reconciliación de dos subprincipios, el de la protección del medio ambiente versus el del desarrollo y aquel principio que los concilia es el del "desarrollo sustentable". ${ }^{15}$ Desarrollo incluye el desarrollo en sí y el beneficio económico producido sino el crecimiento de la felicidad y calidad de vida humana. Cita el autor el concepto de una

Sri Lanka Guardian, "Sri Lanka: Justice C. G. Weeramantry No More”, Sri Lanka Guardian, 6 de enero de 2017. https://www.slguardian.org/2017/01/justice-c-g-weeramantry-no-more/

8 International Court of Justice, Dissenting Opinion of Judge Weeramantry in Legality of the threat or use of nuclear weapons, (Corte Internacional de Justicia), (La Haya: 1996), 433/211.

9 Sergio Peña Neira, "Los Derechos humanos y el aporte de chilenos a los Derechos económicos, sociales y culturales, civiles y políticos", en Anuario de Filosofía jurídica y social "Tóvenes autores chilenos". Agustín Squella (Valparaíso-Santiago: Sociedad chilena de Filosofía jurídica y Social, 2010), 298.

10 International Court of Justice, Dissenting Opinion of Judge Weramanty in the case Gabčíkovo-Nagymaros Project (Hungary/Slovakia), (Corte Internacional de Justicia), (La Haya: 1997), 88/85.

11 International Court of Justice, Dissenting Opinion..., 88/85.

12 International Court of Justice, Dissenting Opinion..., 88/85.

13 International Court of Justice, Dissenting Opinion..., 88/85.

14 International Court of Justice, Dissenting Opinion..., 89/86 par. 5.

15 International Court of Justice, Dissenting Opinion..., 90/87

Número de página no utilizable para citar 
In Memoriam, HE Professor Dr. Christopher Gregory Weeramantry Sergio Peña Neira

"distribución justa de los beneficios que resultan del mismo" del preámbulo de la Declaración del Derecho al Desarrollo. ${ }^{16}$ Sin embargo, el autor expone el sistema de su país, Sri Lanka, una isla, donde por medios jurídicos se mantenía el equilibrio entre la utilización de ciertos recursos (agua, en el ejemplo), versus la necesidad de preservar los mismos. ${ }^{17} \mathrm{El}$ autor indica a la misma como una "civilización" con relaciones exteriores e internacionales extendidas. ${ }^{18}$ Incluso descrito por A. Toynbee. ${ }^{19}$ La idea o el concepto es que recursos naturales, en la especie, renovables, no pueden perderse sin que sea beneficiosos a los seres humanos y demás criaturas vivientes. En este caso, a los habitantes de Sri Lanka. ${ }^{20}$ Estas formas de ver el mundo, el beneficio para los habitantes de los recursos naturales, en el caso de carácter renovable, no es desconocido en Europa. ${ }^{21}$ Es más, este principio se refiere de manera concreta, según el autor, dentro de lo que es la costumbre internacional. ${ }^{22}$

Recibió, además, una serie de premios tanto de su país natal como de organizaciones internacionales de gran prestigio académico así como doctorados honoris causae de universidades de alto prestigio internacional.

Asimismo, fue Presidente de la Asociación Internacional de abogados contra las armas nucleares, fundado en Sri Lanka del Centro Internacional Weeramantry para la Paz, la Educación e Investigación.

Lo sobreviven además de su esposa, cinco hijos y once nietos.

\section{Bibliografía del Profesor Weeramantry (no exhaustiva)}

1. - The law in crisis: bridges of understanding (London: Capemoss, 1975)

2. - Equality and freedom: some third world perspectives (Colombo: Hansa Publishers, 1976)

3. - An invitation to the Law (Sydney: Butterworths, 1982)

4. - Law: The Threatened Peripheries (Colombo: Lake House Investments, 1984)

5. - Islamic Jurisprudence: An international perspective (Houndmills: MacMillan, 1988)

\footnotetext{
International Court of Justice, Dissenting Opinion..., 91/88 nota 3 in fine.

7 International Court of Justice, Dissenting Opinion..., 98/95.

International Court of Justice, Dissenting Opinion..., 98/95, nota 24.

International Court of Justice, Dissenting Opinion..., 99/96.

International Court of Justice, Dissenting Opinion..., 101/98, notas 37 y 38.

1 John Parotta, et al., Traditional knowledge for sustainable forest management and provision of ecosystem services, International Journal of Biodiversity Science, Ecosystem Services and Management, 12: 1-2, 1-4, , DOI: 10.1080/21513732.2016.1169580.

22 Dire Tladi, Sustainable Development in International Law: An analysis of key enviro-economic instruments (Pretoria: Pretoria University Law Press, 2007), 97.
}

Número de página no utilizable para citar 
6. - Human rights and scientific and technological development: Studies on the affirmative use of science and technology for the furtherance of human rights (Tokyo: United Nations University, 1990)

7. - Nauru: environmental damage under international trusteeship (Melbourne; Oxford: Oxford University Press, 1992)

8.- The Impact of technology on human rights: global case-studies: studies on the affirmative use of science and technology for the furtherance of human rights, commissioned as a special project by the United Nations University,

following a reference to the University by the United Nations Human Rights Commission (Tokyo: United Nations University Press, 1993)

9. - Justice without frontiers: furthering human rights (La Haya; Boston: Kluwer Law International, 1997)

10. - Nuclear weapons and scientific responsibility (Ratmalana: Sarvodaya Vishva Lekha; Cambridge, MA, EE.UU.: Kluwer Law International, 1999)

11. - Universalising International Law (La Haya, Brill-Nijhoff, 2004)

12. - A call for national reawakening (Pannipitiya: Stamford Lake, 2005)

13. - Sustainable Justice: reconciling economic, social and environmental law (Leiden; Boston: Martinus Nijhoff Publishers, 2005)

\section{Bibliografía acerca de CG Weeramantry (no exhaustiva)}

Anghie, Antony y STURGess, Garry (eds.), Legal visions of the $21^{\text {st }}$ Century: Essays in honour of Judge Christopher Weeramantry (La Haya; Londres; Boston: Kluwer Law International, 1998) 